\title{
Erratum: Radiation of a charge in dielectric concentrator for Cherenkov radiation: Off-axis charge motion [Phys. Rev. Accel. Beams 22, 083001 (2019)]
}

\author{
Sergey N. Galyamin $\odot$, Viktor V. Vorobev, and Andrey V. Tyukhtin
}

Q (Received 9 September 2019; published 10 October 2019)

DOI: 10.1103/PhysRevAccelBeams.22.109901

The paper contained two mistakes: a minus sign is missed in Eq. (B22) and the plus sign should be replaced by a minus sign in Eq. (B23) of the Appendix B. The correct formulas are given below:
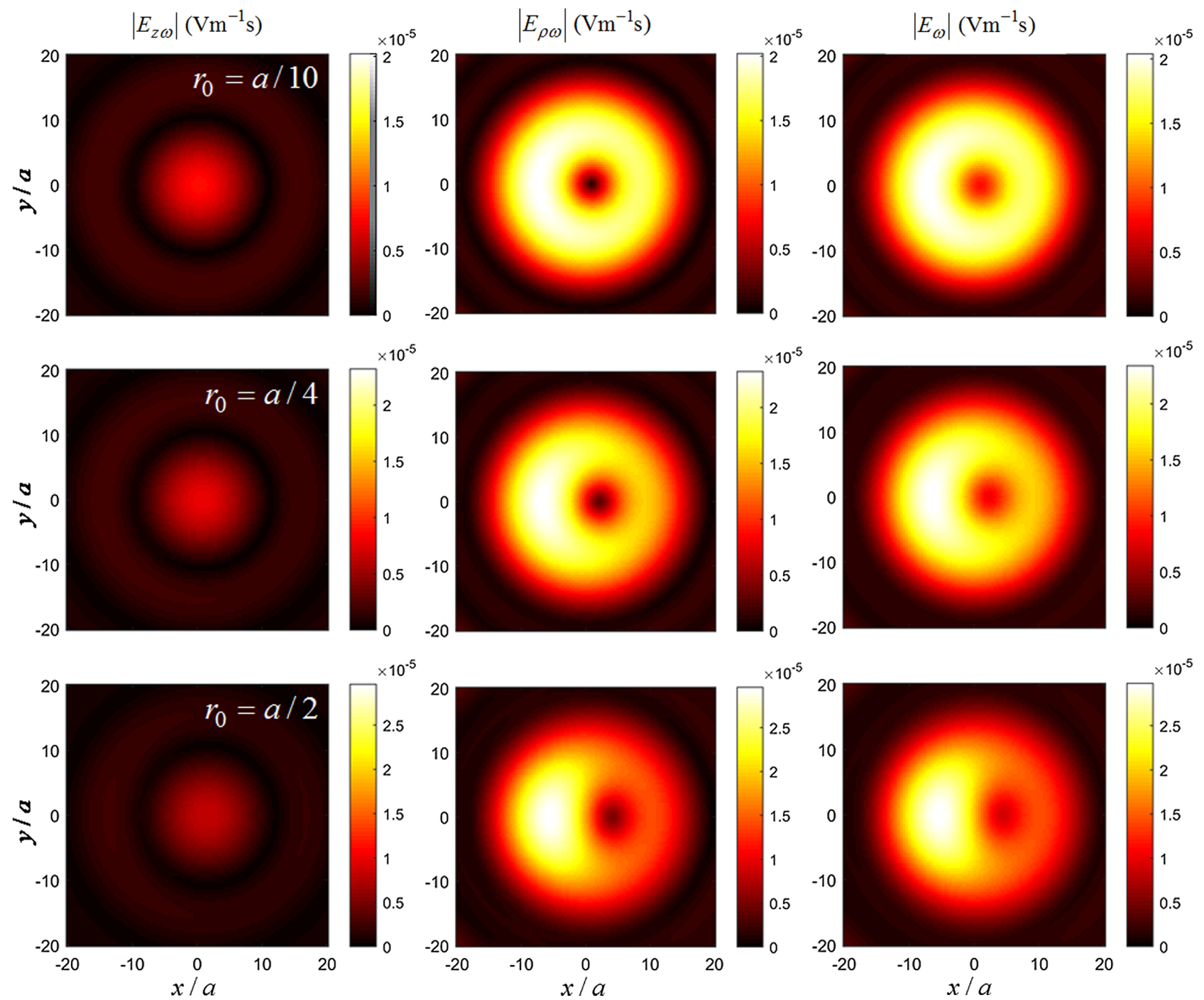

FIG. 1. Two dimensional distribution of absolute values of longitudinal $\left(E_{z \omega}\right)$ and transverse $\left(E_{\rho \omega}\right)$ and total field over $x y$-plane in the focal plane of the concentrator $(z=0)$ for three values of charge's shift $r_{0}=a / 10, a / 4, a / 2$. Calculation parameters are given in the paper.

Published by the American Physical Society under the terms of the Creative Commons Attribution 4.0 International license. Further distribution of this work must maintain attribution to the author(s) and the published articles title, journal citation, and DOI. 


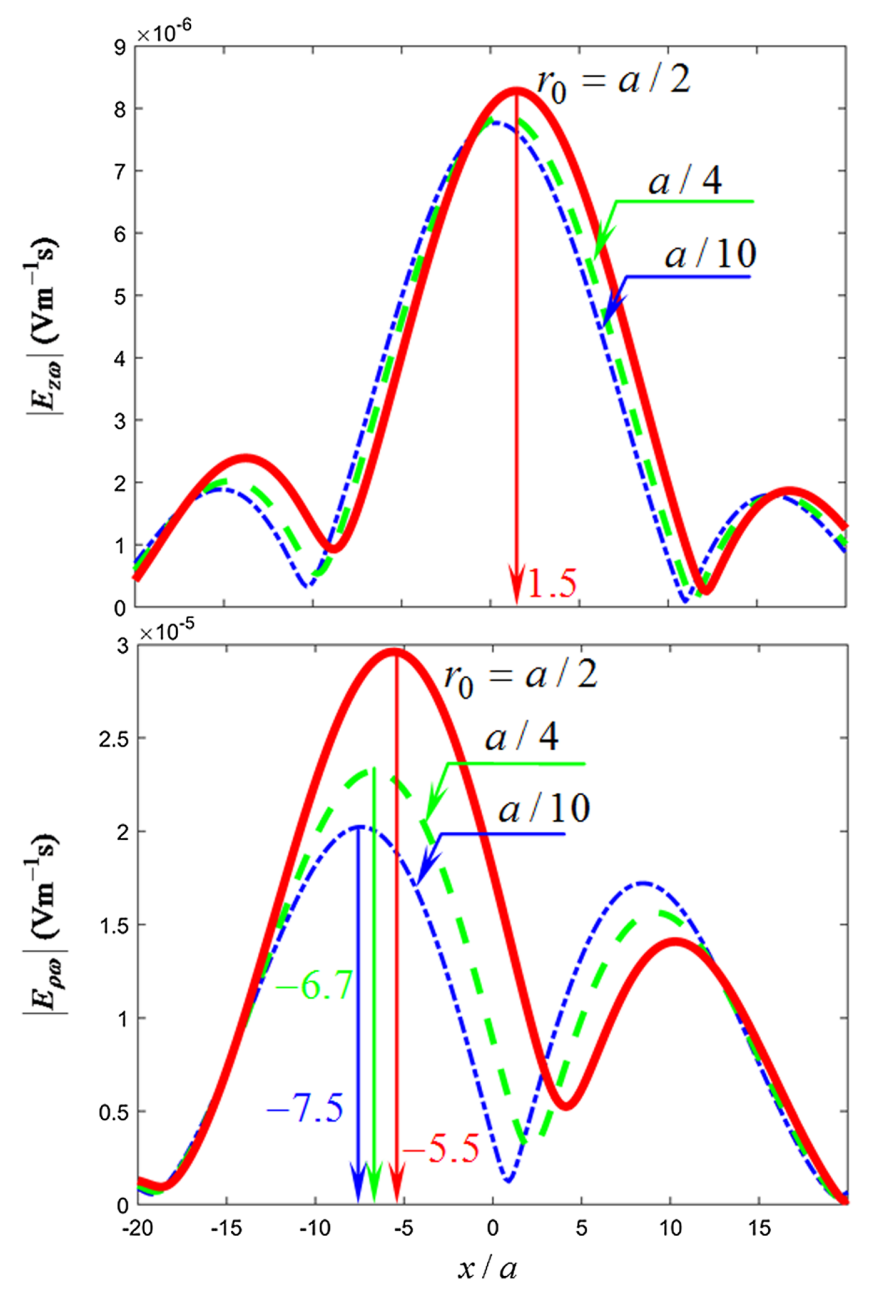

FIG. 2. Dependence of absolute values of longitudinal $\left(E_{z \omega}\right)$ and transverse $\left(E_{\rho \omega}\right)$ field over $x$ for $y=z=0$ for three values of charge's shift $r_{0}=a / 10, a / 4, a / 2$. Calculation parameters are the same as in Fig. 1.

$$
\begin{gathered}
\tilde{A}_{\nu}^{(E 1)}=\frac{1}{\Delta_{\nu} H_{\nu}^{2}}\left\{-\left[\nu(\beta a)^{-1} I_{\nu}\left(\sigma_{0}^{2}+s^{2}\right)\right]^{2} H_{\nu}^{2} K_{\nu} I_{\nu}^{-1}+\left[\sigma_{0}^{2} s \mu H_{\nu}^{\prime} I_{\nu}+s^{2} \sigma_{0} I_{\nu}^{\prime} H_{\nu}\right]\left[\sigma_{0}^{2} s \varepsilon H_{\nu}^{\prime} K_{\nu}+s^{2} \sigma_{0} K_{\nu}^{\prime} H_{\nu}\right]\right\}, \\
\tilde{A}_{\nu}^{(H 1)}=\frac{\nu I_{\nu}\left(\sigma_{0}^{2}+s^{2}\right)}{i \beta a \Delta_{\nu} H_{\nu}}\left\{\left[\sigma_{0}^{2} s \varepsilon H_{\nu}^{\prime} K_{\nu}+s^{2} \sigma_{0} K_{\nu}^{\prime} H_{\nu}\right]-K_{\nu} I_{\nu}^{-1}\left[\sigma_{0}^{2} s \varepsilon H_{\nu}^{\prime} I_{\nu}+s^{2} \sigma_{0} I_{\nu}^{\prime} H_{\nu}\right]\right\} .
\end{gathered}
$$

These small corrections affect the numerical results of the paper for the nonsymmetrical case presented in Figs. 4 and 5. Recalculated figures (Fig. 1 and Fig. 2, respectively) and revised discussion are presented below.

First, asymmetry in the longitudinal field is very weak (see Fig. 1, left column and Fig. 2, top plot). The peak is slightly shifted in the area $x>0$, i.e., in the area where the shifted charge moves, but even for the relatively large offset $\left(r_{0}=a / 2\right)$ the peak shift is only about $1.5 a$. The magnitude of this peak is practically not changed with $r_{0}$, thus the longitudinal field is principally the same as in the symmetrical case for the used parameters.

In turn, the transverse field (see Fig. 1, second column and Fig. 2, bottom plot) has an essential asymmetry: an expressed peak located in the region $x<0$ can be clearly recognized especially for a large offset. The magnitude of this peak increases essentially with an increase in ratio $r_{0} / a$. The shift of this peak is relatively large compared to $a$ and slightly changes with $r_{0}$. Therefore, one can conclude that asymmetry in the transverse field increases with an increase in value of charge shift. Moreover, as one can see from Fig. 2, separate detection of the peaks of the longitudinal field and the transverse field is problematic. For small offsets $\left(r_{0} \lesssim a / 4\right)$ the peak of the longitudinal field is located close to the charge trajectory (i.e., for $\rho<a)$ while for larger offsets $\left(r_{0} \sim a / 2\right)$ it is dominated by a strong transverse field.

Discussion on possible applications of the presented dielectric concentrator should be also revised. First, the longitudinal peak actually has a single peak (instead of two); therefore all statements concerning possible detection of two peaks should 
be removed. However, the strong peak of the transverse field can be actually utilized for determination of beam shift and positioning of the beam toward the axis of the structure. Moreover, this can be done for arbitrary offsets (not only small offsets as was initially stated). The statement that "if the bunch is well aligned along the axis" the "interaction between the bunch and strongly concentrated radiated field can lead to longitudinal modulation of the bunch" can be additionally clarified. As one can see from Figs. 1 and 2, for offsets $r_{0} \lesssim a / 10$ the transverse field is small comparable with the longitudinal field for $\rho<a$. Therefore mentioned modulation is potentially possible for $r_{0} \lesssim a / 10$.

We thank S. Baturin for his interest in the paper and for fruitful discussions, which have helped us to discover the aforementioned mistakes. 